\title{
Conceptual Model of Successful Educational Online Community of Practice for Kingdom of Saudi Arabia
}

\author{
http://dx.doi.org/10.3991/ijet.v9i9.3864 \\ F. Al-Shahrani, H. Mohammad \\ Al Imam Mohammad Ibn Saud Islamic University (IMSIU), Riyadh, Saudi Arabia
}

\begin{abstract}
The community of practice is an internet application that nourishes knowledge management and innovation. Implementing the community of practice in education can enhance the learning process and make it an enjoyable, exiting and lifelong experience. In this paper, the researchers intend to create a conceptual model for the successful implementation of community of practice in Education in the Kingdom of Saudi Arabia through evaluate the literature review about the educational community of practice. Then, a prototype is developed followed by a survey to collect feedbacks about the experiment. Using the Chi-Square statistical method of qualitative analysis associated with the Design Science Methodology of research, the research is fulfilled. The results illustrated the conceptual model of the effective implementation of the community of practice in the Kingdom of Saudi Arabia, that define the seven factors to be considered which are network density, culture perceptions, distributed production, knowledge complexity and richness, government enforcement, IT specialist role, and senior members role.
\end{abstract}

Index Terms-Online Communities of Practices, Communities of practices, Technological context, and Knowledge management

\section{INTRODUCTION}

In this era, using the internet and more specifically the Web, information exchanging and knowledge spreading are convenient advantages. The web becomes a media for searching, communication and collaboration among professional as well as home users. Using the web technologies for educational purposes has enabled to store, exchange and communicate huge educational resources including textbooks, teaching aids, videos, audios, presentation slides, learning objects, teaching aids, worksheets and assignments [1]. After the arrival of Web 2.0, the use of the web technologies is increased. The principles of Web 2.0 are using the web as a stage, attaching collective intellect, and amusing users' experiences. An example of this is Wiki Web Sites. Afterwards, the collaborative learning style becomes an urgent need. It focuses on sharing the educational material, communicate about them and contribute to enhance and add more values to them [2]. Since 2005, the University of Puerto Rico's Río Piedras, The Faculty of the Graduate School of Information Sciences and Technologies is an early adopter of distance education on Campus. They designed numerous online courses as part of a pilot project. The evaluation of the experience showed improvements. It is considered one of the first learning communities of practice that promoted by the social and knowledge learning [2].

In 2006, a program is designed to appeal students interested in careers of learning engineering. The main goals of the program are to help each student to generate new knowledge, correctly apply their knowledge in problem solving, and to transform the new understandings through active and continued participation in the profession. The results showed that there is a deep connection between identity and participation in a professional community of practice [3].

During 2009, the related works about the online communities are connected and three guidelines for supporting online communities of practice are newly proposed. The first guideline is to invest the relationships among homogeneous community. It is critical for maintaining feedback between community end users and designers. This type of communication is considered as a social support or resources. Consequently, it is essential to keep the community moving forward, improving its offerings and growing at the same time. The second guideline is to provide multiple online gathering places for communication among a range of community end users. On the Internet, a gathering place can be a virtual world, a mailing list, a chat room, a blog ... etc. The third guideline is to reinforce leadership roles from within the community. Community leaders perform shaping, governance, interacting and other social support services for the community. Ref. [4] was another important study created a trust model to encourage the reuse of knowledge in Community of Practice. The main feature of this model is that when the employees are rewarded for their contribution of knowledge in the community, this helps in detecting and solving problems in companies or communities [5].

During 2010, a case study of online community of practice is done in the UK. First, it tracks how the online community of practice has developed. Then, it detects the functionality, user types, the used governance methods, and the selected technology. Finally, the benefits it brought to its members is presented. To measure the community of practice's performance, a tool is established and validated [1]. The measuring tool provides an indication of how a community of practice is functioning at a certain moment. The results showed that there is no valid evidence that knowledge management involvements have a positive effect on a community of practice. Therefore, more validation is needed [6].

In the same year, eLearning-based training system is evaluated. The evaluation concluded that eLearning-based 
PAPER

Conceptual Model of Successful Educational Online Community of Practice for ...

training system could improve staff performance as well as the processes of knowledge development within companies. One condition is that to be ready to use eLearning is to have workable training strategies supported by suitable organizational, technical and human resources oriented measures. In addition, social practices like Community of practices are useful for keeping experts and clients in touch as well as for improving knowledge of their members [7].

\section{SIGNIFICANCE OF STUDY}

This study is about the successful implementation of the community of practice in education in the Kingdom of Saudi Arabia. There are plenty of researches about the community of practice in general and the use of the community of practice in education internationally. This research is considered one of the first group of researches that discuss the implementation of educational community of practice in KSA. Moreover, this study is considered one of the first researches about educational community of practice in the gulf and the Arabian countries. It can be considered as a milestone that other Saudi, Gulf and Islamic researches may expand.

\section{LITERATURE REVIEW}

In this section, I will present important related researches to get a full understanding of the community of practice (CoP) and its associated effects. The aim of this is to evaluate the agreed on guidelines, factors, methods and models in order to study them to be applied in the educational community of practice in the Kingdom of Saudi Arabia. Literature review about Community of Practice according to scope of research is divided into 2 major sections. They are literature review and related work

\section{A. literature review}

\section{1) Online CoP Definition \& Context}

Community of Practice (CoPs) are social gatherings that share an interest and possess a sense of belonging that is shared by the members of the CoPs. The aim for designing and developing the CoP is to enhance communications for the sake of creation and innovation. CoPs are expected to promote knowledge management that include knowledge sharing, use, production and collaboration. CoPs have functional objectives, which is to practice. This practice goal involves engagement of people to do actions, and sharing resources, such as procedures, schedules, tools, stories, notions, and artifacts. Online CoP is a group of relations among some people, doing some activity, spread in the whole world during undefined time span. CoPs provide open connections among physically spread participants. In this research $\mathrm{CoP}$ always define the Online $\mathrm{CoP}$ practice and the researchers will refer to it as CoP. Concerning the context of CoPs it is illustrated in figure 1 . The contexts include the knowledge, technology and people, in other words social [8].

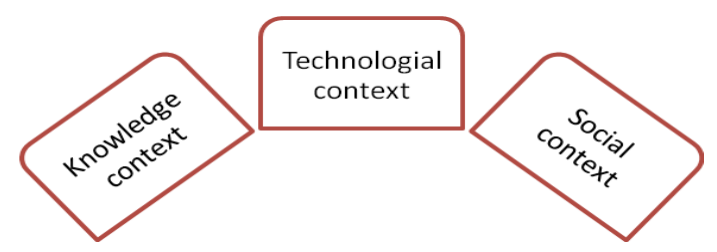

Figure 1. Community of practice context
The knowledge context includes two important aspects. The first aspect is transforming from information sharing to knowledge management that encamps sharing knowledge, using this knowledge, and evolution of knowledge to get new ideas and thoughts. The second factor is the existence of knowledge expertise members. The social context focuses on making the community members act as social knowledge workers to sustain social communication. Finally, the technical context discusses features of online media and technology literacy [8].

\section{B. Case Studies of CoP}

Virtual knowledge communities (VKCs), such as online CoPs, are exceptional knowledge resources that are cheap to use. The focus of (Shin \& Kook, 2010) study is to maximize the use of VKCs. The results stated that knowledge is distributed efficiently when knowledge agents are consistently distributed across the network. The results of this study help understand the inter-community knowledge transfer dynamics in virtual knowledge community networks [9].

The successful implementation of an online CoP contributes innovation and knowledge transfer (KT) application. Online CoPs and Web 2.0 technologies can facilitate participation and improve connectivity within digital networks of knowledge transfer and innovation professionals. The success of social networks is created on the ability to engage a wide range of users and reach serious frame. The principle of reaching large number of users is also vital to professional e-communities [1].

There are three kinds of roles in the CoPs: the newcomer, the coordinator and the core member. Newcomers need time and space to participate. To reach a large number of people imposes appropriate participation of the newcomers. Thus, the factors that may eliminate newcomer's appropriate participation must be studied. In addition, the factors that support newcomers' acceptance in the community of practice are to be clarified too. To support the newcomers' acceptance, some guidelines are proposed.

- Rank status is a negative factor in CoPs because it separates the newcomer from the expert of high rank member.

- The senior partner plays an important role, who can accelerate or slow down the time for acceptance. If the newcomer spends the most time with the senior partner, their legitimacy increased.

- It is necessary to build a democratic culture in CoPs so that the senior member can accept the newcomers easily.

- CoPs must allow newcomers to make mistakes to encourage them to participate.

- Removing economic and safety concerns, which are cultural factors, the member can be less hostile to accept the newcomers [10].

To improvement knowledge in companies, a strategic tactic of organizing eLearning based training is established. Firstly, an assessment of companies' readiness for eLearning and knowledge development took place. Then, the use of Communities of Practice (CoPs) to support learning, sharing, transfer and development of knowledge is observed. The results showed that for companies to be ready to use eLearning as a supportable training strategy, reinforcement by organization, technical and human re- 
PAPER

Conceptual Model of Successful Educational Online Community of Practice for ...

sources is necessary. CoPs are useful by keeping experts and clients in touch as well as for improving knowledge $[7]$.

\section{Related Work}

The context of CoP includes the knowledge, technology and people, in other words social. According to [8], there are inter-relationships among online community's culture, group unity and knowledge sharing. The culture of the online community has direct positive impact on group cohesion. However, it has no direct positive impact on knowledge sharing. Group cohesion has a midway role between the culture of the online community on one side and knowledge sharing on the other side. Good online community culture will increase community members' knowledge sharing intent indirectly, through strong group cohesion. Moreover, online community's managers should pay full attention to group cohesion building up to provide various and huge knowledgebase to make the community more attractive and make members anxious and loyal to share [11].

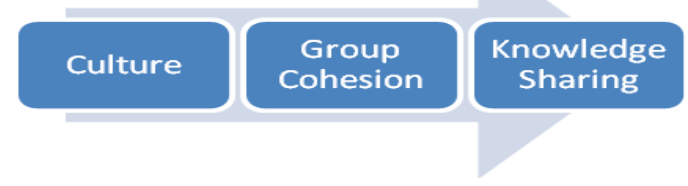

Figure 2. the effect of culture on knowledge sharing

In addition, CoPsare improved by the feeling of trust. CoP's members, that trust each other, can share and reuse the knowledge. The authors of [5] created a trust model to encourage the reuse of knowledge in CoPs in organizations. The proposed model enlists that rewarding the employees if they contribute with knowledge in the community, helps to detect problems in companies. Moreover, the authors created a tool to measure trust that has simple and easy to understand formulas. As in [5], Individuals in online CoPs are vague and the social relationships in the online CoPs are built on computer-based interactions. Consequently, the feelings of trust among the online CoPs' member encourage inspiration, engagement and association [12].

The feedbacks among the members of the CoP, have major impact on members' participations and the overall performance of CoPs. Reputation mechanisms on community knowledge performance are studied using an agentbased simulation and a factorial experiment. The results showed that the reputation mechanisms have a major negative effect on community knowledge performance. Moreover, complex knowledge harmfully affects community outcomes. However, greater network density advances the CoPs [13].

A core matter in designing and emerging community computing infrastructures is the underlying social technical background. Many online communities of practice fail because the fundamental infrastructure does not meet end user necessities. Consequently, the virtual community is incapable to hold the users constantly over time. There has been more than nine years of designing online community of practice for education professionals called Tapped In. After that, the research proposed three design strategies that connect the theoretical and empirical literature in community computing. First, focusing on the rela- tionships among a similar community. This relationship among the end users and the developers of the online community infrastructure is to ensure that the end users' objectives are met.

Second, it is necessary to keep the community refining and expanding. In order to achieve this, there must have settled gathering places. For online communities, gathering place can be a chat room, mailing list, a blog... etc. Gathering places feed relationships, develop an intellect of community, and encourage social interactions. For online communities, participants can be new ones or core members. The key feature is to provide different gathering places for the different types of end users.

A third design strategy is to emphasize leadership roles progressively from within the community. Community leaders implement unifying, authority, interacting, and other social support. The findings included more details. First in order to gain trust of end users, the community developers must respectfully respond to users who submit a bug, complaint, or suggestion. In addition, if the issue is not handled they must explain why. Second, there is always a tension between what the users want and what the developers prioritize. Therefore, it is easy to ignore the needs of the community by the developers. To overcome this problem one of the team members becomes the user ambassador who represent the opinion of the community in all design and development negotiations.

Finally, the research emphasized the value of the developers' constant attempts to participate in each online group or activity. Moreover, they need to understand what is truly important to the community by interacting with the members on their terms [4].

To observe how comments and feedback streamed within an online learning community, a case study of three student teachers is made. In this study, an online digital video database is used as learning object to help to make variation of teaching practices. The teachers were sharing teaching observes during their first teaching year in the teacher education program. The findings concluded that there is a lack of a standardized teaching resources format and well-developed categorization and searching engine. Consequently, users face complications in accessing and sharing online resources. Ultimately, the community users suffer tiresome administrative procedures before uploading resources to a central unit, to ensure efficient management. Moreover, users to produce and save resources in various formats with no attention that of issues such as cross- platform digital sharing [14].

Knowledge sharing through the CoPs' activities that occur among doctors has a great assessment and assistances in improving the quality of medical dealing. The findings of this research showed that the success factors of knowledge-sharing activities are the quality of communication among doctors. Other important factors to consider are reputation, trust, shared goals, moderator role, attitudes such as enjoy Helping, supposed worth and community leaders [15].

\section{The COMmunities OF PRACTICES In KSA}

There are almost no previous researches on The Communities of Practices to address Kingdom of Saudi Arabia in specific. Therefore, I will illustrate a number of researches made in the Kingdom of Saudi Arabia that 
canpave the way to implement the communities of practice in the Kingdom of Saudi Arabia.

On 2008, a group of female researchers at King Saud University has developed and deployed an Arabic learning repository called Marfiah. The project provides an Arabic educational repository to the community for free. Services of the Marfiah project included knowledge sharing by linking to a web page or by uploading educational Arabic content directly to the repository. The learning content can be tracked and rated as well. This example of knowledge sharing can pave the way for the Communities of Practices in the Kingdom of Saudi Arabia. However, it was forsaken and had a little number of users. The two reasons are the absence of consciousness of the worth of such technologies and the absence of advertisement and supporting board [16].

On 2011, a study is made to investigate the mobilelearning acceptance in the Saudi Arabian university. More than $75 \%$ students showed confident attitudes towards mobile -learning. The reasons are the elasticity of learning methods and timings, and enhanced communications among learners [17].

On 2012, another study investigated the same subject matter in Saudi Arabia that is to examine acceptance in mobile learning (m-Learning) but this time to serve in the development of distance learning for higher education. The results from statistical analysis showed that the acceptance level of students on m-Learning is in a high level. Although, more than half of the students were not used to m-Learning, they had a good attitude with it. Therefore, the university administration should focus on the design of m-Learning system. Good insight and university policy association are two major aspects that lead to success mLearning system [18].

According to the previous literature review, there are some factors that may delay or hinder the implementation of $\mathrm{CoP}$ in KSA. They are illustrated in figure 3

\begin{tabular}{|l|}
\hline University undear objectives and unadequate commitment of adoption of online learning in higher education. \\
\hline The lack of the consciousness of the value of such technologies \\
\hline Instructors concerns of issues such as intellectual property rights and loss of privacy. \\
\hline Complexity of the online environment. \\
\hline The low quality of the Internet speed \\
\hline Poor integration between various separtments \\
\hline Privacy issues. \\
\hline Security issues \\
\hline E-plagiarism \\
\hline
\end{tabular}

Figure 3. Causes of Hindering Online Education Implementation in KSA

\section{The CONCEPTUAL Model of IMPLEMENTING CoP IN EDUCATION IN KSA SUCCESSFULLY}

There is gap in the literature review to study the implementation of the Communities of Practice particularly in the Kingdom of Saudi Arabia. By combining the results of the international studies of the implementation of the Communities of Practice and the concluded information about the Saudi literature review about the online implementation in Education, I come up with conceptual framework that enables the successful implementation of Communities of practice in the Kingdom of Saudi Arabia. This conceptual framework will be tested for validation. According the Related Work and Figure 3 Causes of Hindering Online Education Implementation in KSA, the conceptual model will have one dependent variable, which is the successful implementation of CoP and seven independent variable that affect the $\mathrm{CoP}$ success. These are illustrated in figure 4.

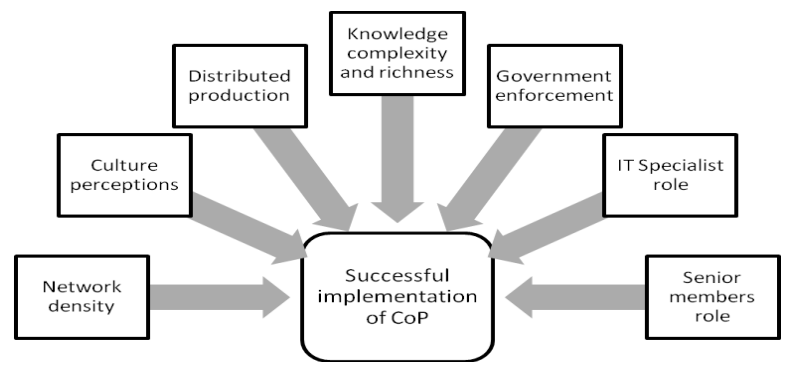

Figure 4. CoP Implementation Conceptual Model

More details of each one are as follow:

1. Network density including different gatherings levels

2. Culture perceptions including trust, privacy, security, newcomer acceptance, discrimination

3. Distributed production including rewarding and rank status

4. Knowledge complexity and richness including latest researches, and latest social news, knowledge presentation and explanation.

5. The Saudi Government enforcement role including strategic goals and policies, moving educational system processes to $\mathrm{CoP}$ as well as to develop national educational $\mathrm{CoP}$

6. IT Specialist role including teaching resources format standardization, categorization, supportive role, leading role, gaining trust

7. Senior members in a $\mathrm{CoP}$ role including interaction enhancement, discipline implementation, communication arrangement, helping role and acceptance of newcomers

The contexts of CoPs are of three main categories knowledge, people and technology [4]. In my conceptual model, knowledge corresponds to the Saudi Educational System. People corresponds to end user and technology corresponds to IT specialists or aware who are specialized in educational applications. Consequently, there are three moderate variables which are the context of Cop. They are the end users, the IT specialists and the Saudi Educational System. These three moderate factors are illustrated in figure 5

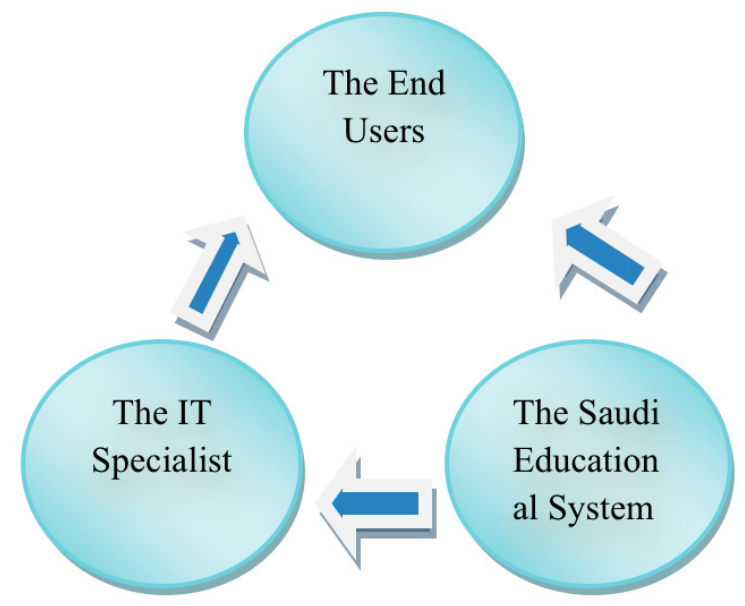

Figure 5. the three main contextual users of the Conceptual Model 


\section{METhODOLOGY}

For this research, I follow the Design Science Framework. The Design Science Research Model (DSR) is appropriate to IS researches because it focuses on the two key issues which are the central role of the IT products as well as the professional relevance of people of IS researches. IT products are solving real-world business problems in a certain context. While, people are the users of these IS products and they have a direct effect on the survival of these IT products [19].

\section{A. Overview on Design Science Methodology}

In order to consider the two factors (IT and Human) there are two methods to adopt Natural Science or (Behavioral Science) and Design Science (March and Smith 1995). The behavioral science is followed to justify theories that clarify or forecast human activities. It developed from natural science research methods. It truly measures the interactions among people, technology, and organizations if an IS is used.

\section{B. Design Science Methodology Guidelines}

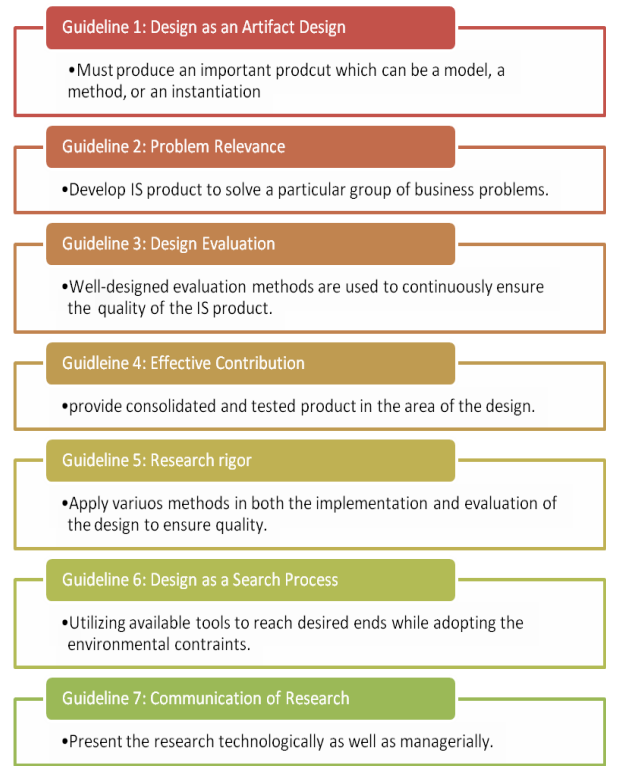

Figure 6. Design Science Framework Guidelines

\section{Design Science Research Cycle}

The Design Science Framework focus on three research cycles which are relevance, rigor and design which are illustrated [19].

\section{1) Relevance Stage:}

The main aim of using this method of research is to produce innovative piece of information related to IS. It captures people, organizations' systems, and technical systems that interact to perform a specific goal. The first step to fulfill this method of research is to identify and introduce gaps of previous researches or problems in an actual application environment.

In this research, the researchers have studied references related to using Cop in organizations, using Cop in Education, factors to make CoP successful, tools to measure CoP Success and more.
Moreover, from the references covered and from the search on the online electronic libraries and databases, it had been found no particular reference about using CoP in Saudi Arabia in Education. Therefore, a gap needs investigation.

\section{2) Rigor Stage:}

In this stage, the latest ideas and frameworks in the same context are defined and used; the aim is to produce specialized ideas about a certain subject matter. Simply, the rigor cycle use past information to the research context to ensure its innovation. To be sure that the latest ideas and frameworks are used to be implemented in Educational CoPs in Saudi Arabia, all of the used references are after 2004. That makes all of the references are done during the last 9 years only.

\section{3) Design Stage}

This stage is the core of the research activities. It includes the repeated steps of checking the IS product, evaluate it, get feedback, and then modify the IS product accordingly. In my own study, the features that enable an effective role of the community of practice in knowledge sharing are proposed in a consolidated framework. A prototype that represents the community of practice for education is developed. After that, surveys are directed based on using the prototype by the participants. Then, the initial conceptual model will be evaluated and adjusted.

\section{Research Method}

This research is a descriptive, interpretative and explanatory. The descriptive aspects help understand the context, in which the $\mathrm{CoP}$ can be implemented in education.

\section{1) Survey}

A self-administered survey is distributed to various types of users. Random sample of educational system members across King Saud University. The users include Stakeholders such as managers and administrators specialized in the education in KSA. Other participants include teachers, professors, and Saudi universities' full-time undergraduate students.

The surveys will use open-ended questions that enable the participants to clarify their points of view about the experience. Surveys open-ended questions have two types. The first type has a number of expected answers. The second type has a wider range of possible answers. The first type had been used.

\section{2) Data Analysis}

The collected data is first stored in an excel file then exported to SPSS 19. For analyzing descriptive categorical qualitative data that comes out of a single sample that has many variables to scale, Chi square analysis is used. Chi square (X2) statistic is used to examine whether circulations of categorical variables fluctuate from one another

\section{RESUlTS AND DISCUSSIONS}

In this section, an overview on the results obtained from SPSS are presented. Then the result for each question of the survey in interpreted. Finally, the overall outcome of the research is illustrated according to the research questions. 


\section{A. Sample Details}

We have 112 particispants including 37 IT specialists, 17 stakeholders or equivalent, and 57 participants interested in education

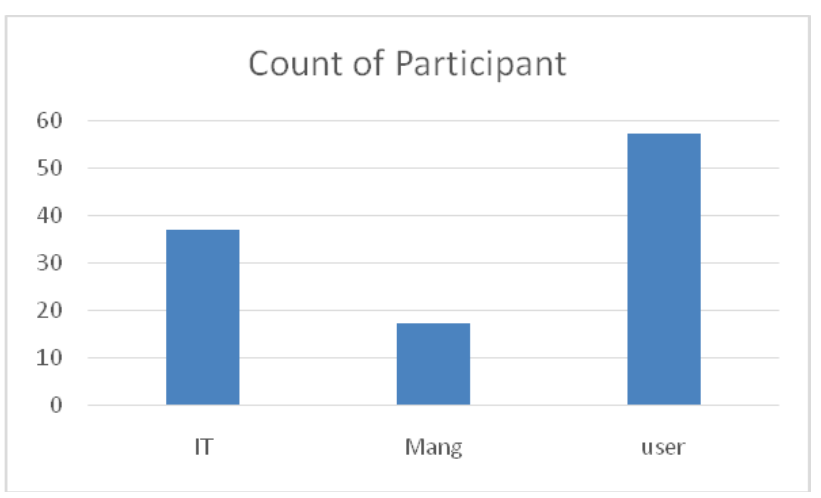

Figure 7. Count and categorizing of participant

Chi-square test results include Case Processing Summary, the inferential statistics, and the descriptive statistics

\section{B. Inferential and the Descriptive Statistics.}

The Chi-square shows if there is a relation or dependency between two measured factors (Variable). The descriptive statistics introduces the number of answers for each choice and the total is presented as graphs produced by Microsoft Excel. The following section introduces the results obtained from SPSS for each question of the survey separately.

\section{1) Participant $*$ Trust}

TABLE I

SPSS RESULTS FOR QUESTION

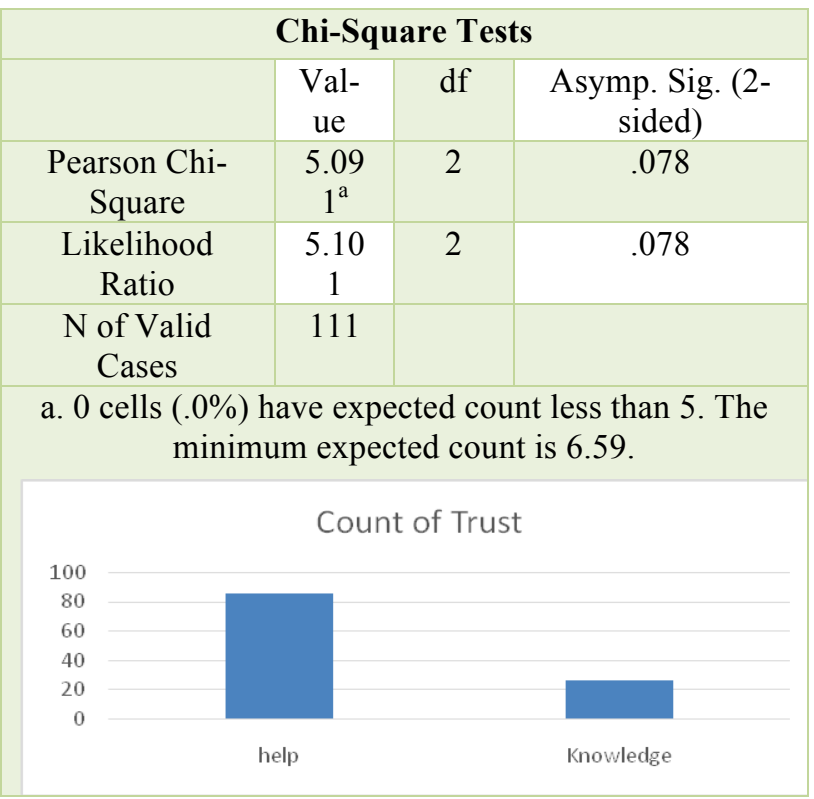

The footnote of the chi-square test is that 0 is below $50 \%$, which means the test is accepted.

In addition, from the Asymp. $\operatorname{Sig}(\mathrm{p})$ we know that $78 \%$ agrees on that trust can empower the CoP. The users illustrated that trust is gained mostly from providing help to other members.
Implementation of CoPs in education in KSA. Trust is developed mainly from members' helping each other. It means trust is an affecting factor on the successful

The final Result for all factors is some of the examined factors proved to be affective and some are not. Accordingly, the proposed conceptual model is modified.

The factors that affect the successful implementation of CoPs are:

1. Network Density:
a. Several gatherings sections of $\mathrm{CoP}$

2. Culture prospective:

a. Trust, which is based on publishing well-known or recent information.

b. Privacy issues which are solved by hiding the real identity of the members.

c. Security

d. Discrimination related to nationality, religion ...etc. effect negatively on newcomer acceptance.

3. Distribution of production

a. Equal distribution of productions \& participations among CoPs members.

4. Knowledge complexity and richness:

a. The level of complexity knowledge

b. Rich knowledge base

c. Good presentation of knowledge

d. Publishing recent researches

5. Saudi Government Role:

a. National enforcement of using educational CoPs which is established by governmental and private sections motivations.

6. IT specialist role:

a. Standardized teaching resources format. In addition, there is a lack of a standardized teaching resources format.

b. Responding to all feedback or technical issues

c. Users' needs can be met by having a developer as users ambassador.

d. IT profession must keep on leading the CoPs.

7. Senior members role:

a. The expected role of the senior members is to provide help, share knowledge and perform corrections for other members

Factors that has no significant association with successful implementation of CoPs:

1. Network Density:

a. The density percentage of members in the CoP

2. Culture prospective:

a. Discrimination related to nationality, religion ...etc. among the $\mathrm{CoP}$ members

3. Distribution of production

a. Rank Status rewarding.

b. CoP excellence rewarding.

c. Prizes encouragements

4. IT specialist role:

a. Categorizing method of the knowledge is NOT an affecting factor on CoPs

5. Senior members role:

a. Senior members affecting newcomers 'acceptance. 


\section{FUTURE WORK AND LIMITATIONS}

During the fulfilment of this research, it was challenging to clarify the different elements that contribute to the success of CoPs. The reason is that in spite the idea is close to our everyday activity using the internet technologies; it relates different and complicated ideas to be understood to be able to create a successful example of CoP.

According to the limitation of time, there are ideas that should be expanded in the future. These ideas include implementing the research experiment on other universities in KSA. The same research needs to be made again but this time to study and implement it in other gulf countries to get results specific for them. Moreover, this research to cover the Arabian countries.

Another future work is supplementing the research with quantitative method of research after modifying the resources of data.

\section{REFERENCES}

[1] Michaelides, R., Tickle, M., \& Morton, S. C. (2010, June). Online communities of practice for innovation and knowledge transfer: A case study in the UK. In Management of Innovation and Technology (ICMIT), 2010 IEEE International Conference on (pp. 922927). IEEE. Retrieved from http://ieeexplore.ieee.org/xpl/ login.jsp?tp=\&arnumber $=5492861 \&$ url $=\mathrm{http} \% 3 \mathrm{~A} \% 2 \mathrm{~F} \% 2 \mathrm{Fieeexp}$ lore.ieee.org\%2Fxpls\%2Fabs all.jsp\%3Farnumber\%3D5492861

[2] Figueras, C. (2005, July). The redesigning of a higher education online course as a virtual learning community: its process and outcomes. In Information Technology Based Higher Education and Training, 2005. ITHET 2005. 6th International Conference on (pp. F4C-1). IEEE

[3] Capobianco, B. M., Diefes-Dux, H., \&Oware, E. (2006, October) Engineering a professional community of practice for graduate students in engineering education. In Frontiers in Education Conference, 36th Annual (pp. 1-5). IEEE http://dx.doi.org/10.1109/ FIE.2006.322517

[4] Farooq, U., Schank, P., Harris, A., Fusco, J. \&Schlager, M. (2007). Sustaining a community computing infrastructure for online teacher professional development: a case study of designing Tapped In.Human-Computer Interaction Series, Springer London. Vol. 16, No. 4-5, pp.: 397-429.

[5] Vizcaíno, A., Portillo-Rodríguez, J., Soto, J. P., \&Piattini, M (2009, February). Encouraging the reuse of knowledge in communities of practice by using a trust model. In Information, Process, and Knowledge Management, 2009. eKNOW'09. International Conference on (pp. 28-33). IEEE. Retrieved from http://ieeexplore.iee.org/xpl/login.jsp?tp=\&arnumber=4782561\& url $=$ http $\% 3 \mathrm{~A} \% 2 \mathrm{~F} \% 2 \mathrm{Fiee}$ xplore.ieee.org $\% 2 \mathrm{Fxpls} \% 2 \mathrm{Fabs}$ all.jsp $\% 3$ Farnumber\%3D4782561

[6] Van der Meijden, M., \& Jansen, S. (2010, May). Performance measuring in Communities of Practice: a method and case study. In Research Challenges in Information Science (RCIS), 2010 Fourth International Conference on (pp. 607-618). IEEE. Retrieved from http://ieeexplore.ieee.org/xpl/login.jsp?tp=\&arnumbe $\mathrm{r}=5507290 \&$ url=http $\% 3 \mathrm{~A} \% 2 \mathrm{~F} \% 2 \mathrm{Fieeexplore.iee.}$ org $\% 2 \mathrm{Fxpls} \% 2$ Fabs_all.jsp\%3Farnumber\%3D5507290

[7] Hamburg, I. (2010, May). eLearning 2.0 and social, practiceoriented communities to improve knowledge in companies. In Internet and Web Applications and Services (ICIW), 2010 Fifth International Conference on (pp. 411-416). IEEE. Retrieved from http://ieeexplore.ieee.org/xpls/abs_all.jsp?arnumber=5476506

[8] Zheng, Y., Li, L., \& Zheng, F. (2010). Context analysis for online communities of nractice. In 2010 2nd International Conference on Education Technology and Computer (Vol. 1).

[9] Shin, S. K., \& Kook, W. (2010, January). Knowledge Transfer in a Multiple Virtual Communities Network. In System Sciences (HICSS), 2010 43rd Hawaii International Conference on (pp. 110). IEEE.
[10] Lihua, L. (2010, May). Support Newcomer's Learning in Communitv of Practice: In Terms of Legitimate Peripheral Participation. In ICEE (pp. 1956-1957).

[11] Chen, M., Zhou, J., \& Zhao, L. (2008, December). The Effect of Virtual Community Culture and Group Cohesion on Knowledge Sharing: A Case Study of Professional Virtual Community. In Computer Science and Software Engineering, 2008 International Conference on. Vol. 5, pp. 105-108. IEEE. Retrieved from http://ieeexplore.ieee.org/xpl/login.jsp?tp=\&arnumber $=4722853 \&$ url $=$ http $\% 3 \mathrm{~A} \% 2 \mathrm{~F} \% 2$ Fieeexplore.ieee.org $\% 2 \mathrm{Fxpls} \% 2 \mathrm{Fabs}$ _all.jsp \%3Farnumber\%3D4722853

[12] Zhao, J., Dong, K., Yu, J., \& Hu, W. (2013, October). DSN: A Knowledge-Based Scholar Networking Practice Towards Research Community. In eScience (eScience), 2013 IEEE 9th International Conference on (pp. 238-244). IEEE. Retrieved from http://ieeexplore.ieee.org/xpl/login.jsp?tp $=\&$ arnumber $=6683913 \&$ url $=$ http $\% 3 \mathrm{~A} \% 2 \mathrm{~F} \% 2$ Fieeexplore.ieee.org $\% 2$ Fxpls $\% 2$ Fabs_all.jsp \%3Farnumber\%3D6683913

[13] Havakhor, T., \&Sabherwal, R. (2013, January). Knowledge Sharing in Peer-to-Peer Online Communities: The Effects of Recommendation Agents and Community Characteristics. In System Sciences (HICSS), 2013 46th Hawaii International Conference on ( $\mathrm{pp}$. 3553-3562). IEEE. Retrieved from http://ieeexplore.ieee.org/xpl/login.jsp?tp=\&arnumber $=6480274 \&$ url=http $\% 3 \mathrm{~A} \% 2 \mathrm{~F} \% 2 \mathrm{Fieeexplore.ieee.org} \% 2 \mathrm{Fxpls} \% 2 \mathrm{Fabs}$ all.jsp $\% 3$ Farnumber\%3D6480274

[14] Landge, P. S., Kamble, V. A., \&Dange, A. S. (2010, July). A virtual learning community for teacher education. In Computer Science and Information Technologv (ICCSIT). 2010 3rd IEEE International Conference on (Vol. 3, pp. 457-460). IEEE.

[15] Firdaus, O. M., Suryadi, K., Govindaraju, R., \& Samadhi, T. A. (2012, June). Knowledge sharing model development in community of practice $(\mathrm{CoP})$ among doctors. In Management of Innovation and Technology (ICMIT), 2012 IEEE International Conference on (pp. 208-213). IEEE. Retrieved from $\mathrm{http}: / /$ ieeexplore.ieee.org/xpl/login.jsp?tp=\&arnumber $=6225806 \&$ url $=$ http $\% 3 \mathrm{~A} \% 2 \mathrm{~F} \% 2 \mathrm{Fiee} x$ lore.ieee.org $\% 2 \mathrm{Fxpls} \% 2 \mathrm{Fabs}$ _all.jsp $\% 3$ Farnumber\%3D6225806

[16] Almalki, A. M. (2011). Blended Learning in Higher Education in Saudi Arabia: A Study of Umm Al-Qura University (Doctoral dissertation, RMIT University).

[17] Chanchary, F. H., \& Islam, S. A. M. I. U. L. (2011). Mobile learning in Saudi Arabia-prospects and challenges. See also website: http://www. nauss. edu. sa/acit/PDFs/f2535. pdf.

[18] Moore, R. W., \&Rajsekar, A. (2010, July). Irods: Data sharing technology integrating communities of practice. In Geoscience and Remote Sensing Symposium (IGARSS), 2010 IEEE International (pp. 1984-1987). IEEE

[19] Fan, S., Zhang, Y., Fan, J., He, Z., \& Chen, Y. (2010, April). The Application of Virtual Reality in Environmental Education: Model Design and Course Construction. In Biomedical Engineering and Commuter Science (ICBECS), 2010 International Conference on (pp. 1-4). IEEE.

\section{AUTHORS}

Fatimah Al-Shahrani is master student, at information system department, Al Imam Mohammad ibn Saud Islamic University (IMSIU), Riyadh (e-mail: fatimah.shahrani@gmail.com).

Heba Mohammad is an Assistant Professor of Information Systems at the College of Computer and Information Sciences. Her research focuses on knowledge management, e-Business, and communities of practice. She also provides different consultation services to various institutions. (e-mail: hkmohammad@ccis.imamu.edu.sa).

Submitted 11 May 2014. Published as resubmitted by the authors 25 October 2014. 